Z Epileptol 2011 · 24:66-66

DOI 10.1007/s10309-010-0162-1

๑) Springer-Verlag 2011
Kommentar zu:

\section{Revidierte Terminologie und Konzepte zur Einteilung von epileptischen Anfällen und Epilepsien}

\author{
Z Epileptol 2010 · 23:66-66 \\ DOI 10.1007/s10309-010-0127-9
}

\section{Leserbrief}

Ich versuche mich gerade mit der revidierten Terminologie und Konzeption von epileptischen Anfällen und Epilepsien auseinander zu setzen. Ihr habt ja in der Zeitschrift für Epileptologie, Heft 4/2010, eine Lanze für sie gebrochen und ich kann mich Eurer Zustimmung durchaus im Prinzip anschließen. Es macht mir nichts aus, statt idiopathisch genetisch und statt kryptogen unbekannt einzusetzen, weiß aber nicht, welche Vorteile sich daraus in der praktischen Arbeit ergeben sollen, wenn doch auch vorher alle wussten, was damit gemeint war. Viel schwieriger und in der Praxis äußerst unbequem zu handhaben, finde ich die Abschaffung des Begriffes „komplex“ (-fokal), den ich in den allermeisten Fällen mindestens so griffig oder nützlich fand wie das von Euch erwähnte „hypermotorisch“. Ich fürchte mich jedenfalls vor bandwurmartigen Diagnosen aus Epilepsiezentren, bei denen versucht wird, die gesamte Semiologie der fokalen Anfälle in die Diagnose zu packen - das fängt ja nach meiner Wahrnehmung durchaus schon an. Es wäre $\mathrm{m}$. E. viel hilfreicher gewesen, eine Kategorie fokaler Anfälle zu schaffen, die sich nicht in einfach oder komplex differenzieren lassen - dann wäre das Problem der manchmal nicht zu leistenden Einordnung auch gelöst gewesen und man hätte trotzdem noch bei „komplex-fokal“ einen kurzen und einprägsamen Begriff gehabt, der sozialmedizinisch äußerst wertvoll verwendet werden kann. Schade finde ich auch, den für mich ebenso griffigen Begriff, ,sekundär generalisiert" jetzt durch „netzwerküberspringend“ oder so ähnlich ersetzen zu müssen - schön klingt das ja nicht gerade, auch wenn es natürlich viel „wissenschaftlicher" ist.

Ihr vermittelt in Eurem Vorwort den Eindruck, als ob diese Revidierte Terminologie und Konzeption zur Einteilung von epileptischen Anfällen und Epilepsien eine beschlossene neue Klassifikation sei. Ich hatte die Autoren der Klassifikationskommission der ILAE eigentlich sowohl bei der Veröffentlichung in der Epilepsia als auch jetzt bei Eurer Übersetzung so verstanden, dass es sich um einen Vorschlag handelt, der in der epileptologischen Weltöffentlichkeit diskutiert (und vielleicht auch ausprobiert) werden soll, bevor er endgültig beschlossen wird. Das müsste dann ja wohl auch der Vorstand der ILAE und nicht die Kommission machen. Entsprechende Auffassungen habe ich auch aus dem Vorstand der Deutschen Gesellschaft für Epileptologie gehört. Vielleicht sollte das noch einmal im nächsten Heft der Zeitschrift für Epileptologie klargestellt werden?

Ihr führt auch aus, dass prognostische Begriffe wie "benigne" gestrichen seien - trotzdem taucht es in der Tabelle 3 der elektroklinischen Syndrome überall wie- der auf (z. B. benigne familiäre neonatale Epilepsie, benigne Epilepsie mit zentrotemporalen Spikes, etc.) - wie ist das zu verstehen?

Sollte es sich bei diesem Vorschlag tatsächlich um eine neue verbindliche Terminologie handeln, so wäre es aus Sicht der niedergelassenen Neurologen und Epileptologen unbedingt erforderlich, den entsprechenden Teil des ICD so schnell wie möglich zu korrigieren, da es einfach keinen Sinn macht, bei den Diagnosen in Arztberichten und Studien andere Begriffe und Einteilungen zu verwenden als bei der Diagnosenverschlüsselung für die Krankenkassen. Hat daran in der Kommission der ILAE überhaupt schon jemand gedacht?

Es ist wahrscheinlich kein Zufall, dass in der Kommission der ILAE kein/e einzige/r deutschsprachige/r Experte/in sitzt, obwohl die Deutsche Sektion die zweitgrößte nach den USA stellt. Es würde der Arroganz der Angelsachsen gewiss keinen Abbruch tun einzuräumen, dass manche Definitionen und Beschreibungen zunächst in anderen Sprachen vielleicht sogar besser zu beschreiben sind als im Englischen. Ich finde das schade.

\section{Korrespondenzadresse \\ Dr. Dieter Dennig}

Neurol. Gemeinschaftspraxis am Seelberg

Seelbergstr. 11, 70372 Stuttgart

ddennig@t-online.de 